\title{
An Olfactory Circuit Increases the Fidelity of Visual Behavior
}

\author{
Dawnis M. Chow, ${ }^{1,3}$ Jamie C. Theobald, ${ }^{4}$ and Mark A. Frye $\mathrm{e}^{1,2,3}$ \\ ${ }^{1}$ Molecular, Cellular and Integrative Physiology Interdepartmental Program, University of California Los Angeles, Los Angeles, California 90095, ${ }^{2} \mathrm{Howard}$ \\ Hughes Medical Institute, ${ }^{3}$ Department of Integrative Biology and Physiology, University of California Los Angeles, Los Angeles, California 90095, and \\ ${ }^{4}$ Department of Biological Sciences, Florida International University, Miami, Florida 33199
}

Multimodal integration allows neural circuits to be activated in a behaviorally context-specific manner. In the case of odor plume tracking by Drosophila, an attractive odorant increases the influence of yaw-optic flow on steering behavior in flight, which enhances visual stability reflexes, resulting in straighter flight trajectories within an odor plume. However, it is not well understood whether contextspecific changes in optomotor behavior are the result of an increased sensitivity to motion inputs (e.g., through increased visual attention) or direct scaling of motor outputs (i.e., increased steering gain). We address this question by examining the optomotor behavior of Drosophila melanogaster in a tethered flight assay and demonstrate that whereas olfactory cues decrease the gain of the optomotor response to sideslip optic flow, they concomitantly increase the gain of the yaw optomotor response by enhancing the animal's ability to follow transient visual perturbations. Furthermore, ablating the mushroom bodies (MBs) of the fly brain via larval hydroxyurea (HU) treatment results in a loss of olfaction-dependent increase in yaw optomotor fidelity. By expressing either tetanus toxin light chain or diphtheria toxin in gal4-defined neural circuits, we were able to replicate the loss of function observed in the HU treatment within the lines expressing broadly in the mushroom bodies, but not within specific mushroom body lobes. Finally, we were able to genetically separate the yaw responses and sideslip responses in our behavioral assay. Together, our results implicate the MBs in a fast-acting, memoryindependent olfactory modification of a visual reflex that is critical for flight control.

\section{Introduction}

The fruit fly combines the perception of visual motion, or optic flow, with olfactory cues, to successfully locate food resources (Chow and Frye, 2009). Optic flow induces a robust motor behavior termed the optomotor response (OMR), whereby the animal steers to minimize retinal slip (Hassenstein and Reichardt, 1956; Tammero et al., 2004). OMRs to visual stimuli such as drifting sine gratings have revealed properties of early visual processing, including the computational structure of the circuit underlying elementary motion detection (Hassenstein and Reichardt, 1956) and the spacing of motion detection (Götz, 1964; Borst et al., 2010). These studies are complemented by electrophysiological investigations in larger flies that characterize the tuning of motion-sensitive cells in the third optic ganglia (Borst et al., 2010). We know significantly less about

Received April 5, 2011; revised July 27, 2011; accepted Aug. 31, 2011.

Author contributions: D.M.C., J.C.T., and M.A.F. designed research; D.M.C. performed research; D.M.C. analyzed data; D.M.C. and M.A.F. wrote the paper.

M.A.F. is an Early Career Scientist with the Howard Hughes Medical Institute. Funding was provided by a National Science Foundation grant (IOS-0718325) to M.A.F. and by the UCLA Dissertation Year Fellowship to D.M.C. We thank Scott Waddell, Leslie Griffith, Justin Slawson, Tim Lebestky, David Krantz, Zhe Zhi Yong, and Bloomington Stock Center for fly stocks. We thank David Krantz for advice on fly strains. We thank Michael Reiser, Tim Lebestky, Jessica Fox, Trista Yip, Jacob Aptekar, and Sara Wasserman for insightful comments.

The authors declare no competing financial interests.

This article is freely available online through the J Neurosci Open Choice option.

Correspondence should be addressed to Mark A. Frye, Department of Integrative Biology and Physiology, University of California Los Angeles, Terasaki Life Sciences, 610 Charles Young Drive East, Los Angeles, CA 90095-7239. E-mail:frye@ucla.edu.

DOI:10.1523/JNEUROSCI.1736-11.2011

Copyright $\odot 2011$ the authors $\quad 0270-6474 / 11 / 3115035-13 \$ 15.00 / 0$ the circuitry for fully transforming visual motion signals into OMRs.

Traditionally, OMRs have been studied with panoramic rotational cues, but flies exhibit stronger OMRs to optic flow generated during sideslip. Sideslip responses, by comparison to rotational yaw responses reach steady state more quickly in time, are differentially sensitive to contrast and spatial extent (Duistermars et al., 2007) and intriguingly, are transformed into motor output faster and more reliably than yaw (Theobald et al., 2010a). The differential spatial, contrast and dynamical responses suggest that independent neural pathways process the sensorimotor transformation of different patterns of optic flow.

Flight OMRs enable stability but are also important for olfactory navigation in flies (Frye et al., 2003). Yaw and sideslip OMRs are differentially modified by olfactory cues (Chow and Frye, 2008). Olfactory-mediated changes in optomotor gain during flight, which we have termed olfactory modification of the optomotor response (OMOR), may comprise a mode switch from variable exploratory flight to reliable tracking flight. Similar mode switching has been observed in the statistical distribution of turns and move lengths during free flight. In the absence of odor, fruit flies are thought to execute saccadic changes in flight direction at a rate optimal for locating distant and spatially unpredictable food resources, but generate turns in a more randomly distributed way upon approaching an odor source (Reynolds and Frye, 2007).

Here we demonstrate that OMOR may be measured quickly and robustly using a previously described white noise analysis technique (Theobald et al., 2010a). We show that olfactory cues increase the fidelity and gain of the yaw optomotor response. By 
contrast to the increase in yaw gain, an attractive odor decreases the gain of sideslip responses. We demonstrate that yaw and sideslip OMRs are separable through genetic inactivation of gal4defined neural circuits. Finally, we provide evidence that OMOR depends on the mushroom bodies (MBs), a neuropil classically associated with olfactory learning and memory. A growing body of literature implicates the MBs in "cognitive" visual behaviors, such as overcoming noise (Xi et al., 2008), visual attention (van Swinderen and Greenspan, 2003), and decision making (Tang and Guo, 2001; Zhang et al., 2007). We make a novel addition by showing that MBs play an important a role in rapid memoryindependent multimodal sensorimotor transformations.

\section{Materials and Methods}

Animal care and preparation. Adult female Canton-S (CS) Drosophila melanogaster (Meigen) maintained on a standard molasses medium in a 12:12 d/night cycle were collected 4-6 d after eclosion. Animals were cold anesthetized using a Peltier device and then attached to a $0.1-\mathrm{mm}-$ diameter tungsten pin with UV-activated windshield glue (Plas-Pak Industries). A burst of UV light from a light-curing gun (ELC-410, Electro-Lite) is sufficient to cure the small drop of glue on the anterior part of the dorsal thorax after annealing. Flies were allowed at least $1 \mathrm{~h}$ to recover before the start of the experiment.

Fly strains. We used UAS lines UAS-TnT to express tetanus toxin light chain $(\mathrm{TeTxLc})$ and UAS-DTI to express diphtheria toxin (DT) (Holmes et al., 2007) as circuit inactivators in combination with gal4 strains. TeTxLc degrades the protein synaptobrevin, preventing synaptic transmission in cells that express it. In contrast, DT is an RNA translational inhibitor that results in cell death. The differences in the function of the two effectors have important implications, as electrical synapses are relatively unaffected by the expression of TeTxLc. Visual interneurons in the third optic ganglia of the Drosophila visual system, the lobula and lobula plate, are known to express electrical gap junctions that heavily influence their physiology (Borst et al., 2010). Furthermore, gap junction mRNA transcripts such as shaking- $B$ are heavily expressed during the development of the adult brain, including possibly in the MBs (Stebbings et al., 2002), and are critical for survival since mutations in the gap junction protein family (innexins) are often lethal.

To target the mushroom bodies, we used the gal-4 lines $17 \mathrm{~d}, \mathrm{c739}$, c305a, 1471, H24, 201y, MB247, c320, OK107, and D52H (Aso et al., 2009). All MB lines were backcrossed to CS for at least seven generations. The MBs are composed of three main lobes, the $\alpha / \beta, \alpha^{\prime} / \beta^{\prime}$, and $\gamma$ lobes, that are thought to have distinct roles in olfactory learning and memory (Davis, 2011). These lobes are generated by four MB neuroblasts that give rise to heterogeneous clonal units. Furthermore, there is evidence that these clonal units receive distinct combinations of $\mathrm{PN}$ output from the antennal lobes (Ito and Awasaki, 2008). Interestingly, the $\alpha / \beta, \alpha^{\prime} / \beta^{\prime}$, and $\gamma$ lobes form sequentially at temporally distinct points during development (Lee et al., 1999). The fly lines targeting the MBs were chosen based their coverage of these MB lobes. Specifically, the lines OK107-gal4 and c320-gal4 target the entire MBs, but also have significant outside expression (Aso et al., 2009). The lines D52H-gal4 and H24-gal4 represent an intermediate level of MB coverage, each expressing in the $\alpha / \beta$ and $\gamma$ lobes (Aso et al., 2009). Last, we chose a number of lines that target a single MB lobe class, including $c 739$-gal 4 and $17 \mathrm{~d}$-gal 4 targeting the $\alpha / \beta$ lobes, c305a-gal4 targeting the $\alpha^{\prime} / \beta^{\prime}$ lobes, and H24-gal4 targeting the $\gamma$ lobes. Furthermore, we used cAMP mutants rut1 and $d n c 1$ (Bloomington), which have defects in short-term olfactory memory. The rutabaga and dunce proteins are primarily expressed in the MBs (Margulies et al., 2005).

We also used the peripheral olfactory line GH298-gal4 to target antennal lobe lateral interneurons also ablated by HU treatment (Stocker et al., 1997) as well as OR lines 22a, 42b, and 43b to target olfactory receptor neurons (ORNs) relevant to the detection of our odorant (Semmelhack and Wang, 2009). Last, we used flies with a well known polymorphism of the foraging allele that has a significant effect on the dispersal rate of Drosophila adults and larvae, such that $R$ variants dispersing much further than $S$ variants (Pereira and Sokolowski, 1993). Our control line was either CS or Population Caged Flies (PCF), a line descended from wildcaught animals in Berkeley, CA (Frye and Dickinson, 2004), as noted in the figures.

Virtual flight arena and odor delivery. The virtual flight arena is described in detail in (Reiser and Dickinson, 2008). Briefly, flies were rigidly suspended in the center of a cylindrical electronic visual flight (Fig. $1 \mathrm{~A}$ ). The arena itself is composed of a $32 \times 88$ pixel computer controlled LED display. Each pixel is spaced $3.75^{\circ}$ from the perspective of the fly, a smaller value than the interommatidial angle of $5^{\circ}$ in Drosophila (Land, 1997). An infrared diode projects light onto the beating wings of the animal, casting a shadow onto an optical sensor below. Custom hardware (wingbeat analyzer, JFI Electronics) extracts the overall wingbeat frequency and individual wing stroke amplitudes at a sample rate of $500 \mathrm{~Hz}$. The steering attempts of the fly may later be reconstructed by subtracting the right amplitude signal from the left, producing a left-minus-right voltage trace $(\Delta \mathrm{LR})$ proportional to the steering torque produced by the animal that comprises the behavioral response metric for our experiments. Custom software written in Matlab (MathWorks) stores the digitized signal. $\Delta$ LR waveforms were processed with a fifth-order zero-phase $200 \mathrm{~Hz}$ low-pass digital Butterworth filter to remove highfrequency noise. The signal from the wingbeat analyzer was also fed back into the display controller to close a feedback loop and allow animals control of the visual stimuli.

During the open-loop portions of the experimental trial, wide-field motion was conveyed using a random cloud of lit pixels, either rotating around the animal about yaw (Fig. 1A) or translating past the animal from a pole of expansion on the fly's right and converging at a pole of contraction on the fly's left to simulate leftward sideslip. The dot cloud motion steps were rendered finer than interommatidial spacing and at faster rates than the animal's flicker fusion frequency, and followed perspective-corrected trajectories to simulate true optic flow (Fig. $1 B$ ). For details on construction of dot cloud stimuli, please see Theobald et al. (2010a). Briefly, each dot cloud had a density of $\sim 16.28 \%$ on the twodimensional display. At the beginning of each stimulus presentation, one of 50 randomly generated dot clouds for each motion type was chosen, to control for any biases due to unique features in each pattern.

Two 20 gauge stainless steel hypodermic tubes adjoined in a plastic pipette tip delivered either water-vapor or apple cider vinegar vapor (Ralph's brand) to the headspace of the animal (Fig. 1C). The air flow was regulated to $40 \mathrm{ml} / \mathrm{min}$ via a mass flow controller (Sable Systems) and directed through a polytetrafluoroethylene test tube containing distilled water (control) or a piece of filter paper soaked in apple cider vinegar. A computer controlled three-way valve switched air flow between the two test tubes. In Figure $2 A$, bottom row, the odor tube was replaced by a secondary water tube as an additional control. To verify accurate placement of the pipette tip, we administered a brief odor pulse and quantified the resultant changes in wingbeat frequency (WBF) and amplitude before each trial. Only animals that responded to the odor pulse with a noticeable increase of either metric were subsequently tested.

Stimulus presentation. The protocol for the virtual flight arena experiment consisted of four presentations of a pseudorandom motion sequence played at $50 \mathrm{~Hz}$ (a randomly shifted 10th- or 11th-order "maximum-length shift register sequence," or m-sequence-see Results section for more detail). The four presentations consisted of each possible combination of visual (yaw and sideslip) and olfactory (water vapor and odor) stimuli. The order of presentation was also randomized at each trial. The first $15 \mathrm{~s}$ of the experiment always consisted of closed-loop stripe fixation, with a $5 \mathrm{~s}$ odor burst in the median $5 \mathrm{~s}$ increment, to allow the assessment of any changes in wingbeat frequency due to odor. Furthermore, each stimulus presentation was interspersed with $5 \mathrm{~s}$ of closedloop stripe fixation to motivate the animal throughout the experiment. Any individual presentation in which the fly stopped flying for $>5 \mathrm{~s}$ was excluded from the analysis. Between $10 \%$ and $30 \%$ of wild-type animals (depending on olfactory condition and visual context) were excluded from analysis based on this criterion.

Hydroxyurea treatment. Hydroxyurea (HU) is a DNA synthesis inhibitor that has been shown to effectively prevent the development of the mushroom body neuropil during the first $5 \mathrm{~h}$ of larval life in Drosophila melanogaster (Prokop and Technau, 1994). Media containing hy- 
A

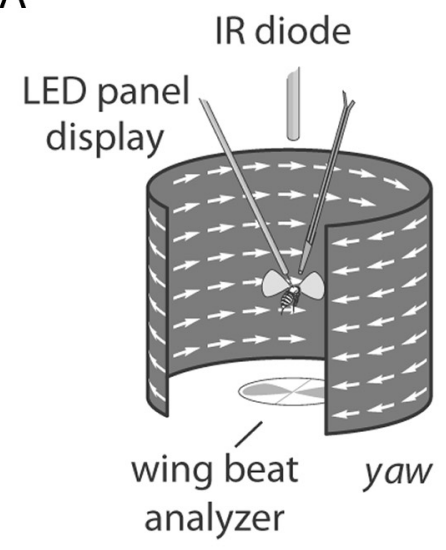

D

linear systems analysis

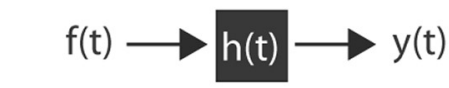

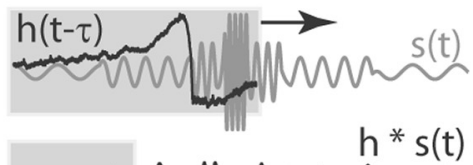

AnAmanminam

$\mathrm{h}=$ linear filter

$s=$ stimulus

$h{ }^{*} s(t)=$ linear prediction

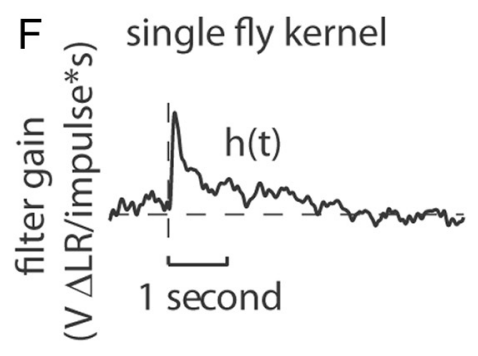

B

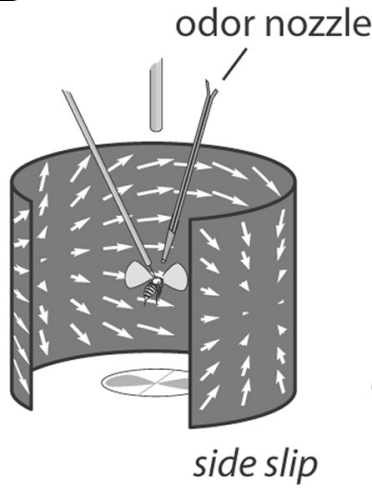

E

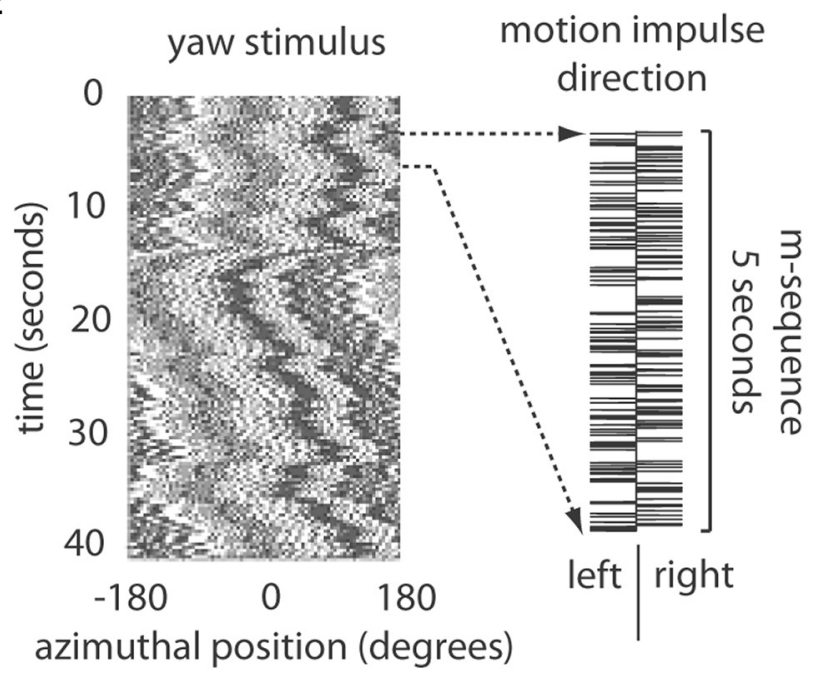

G

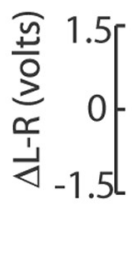

C

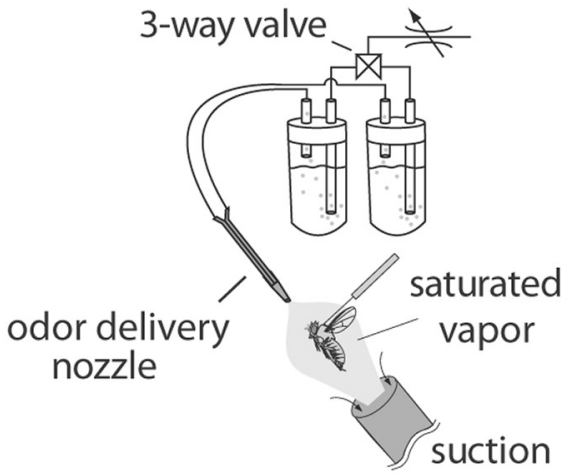

mass flow controller 
tive number of flies in the water vapor side of the trap, and 50 was the number of flies in each replicate. The assay is "free choice" in that flies may elect whether or not to enter any trap. In that respect, the response index will be positive if flies prefer the odor trap, negative if the flies prefer the water trap, or zero if either the preference is balanced or flies do not enter the traps. However, at the end of the $24 \mathrm{~h}$ period, $<2 \%$ of the animals remained untrapped.

Analysis. To obtain the individual impulse response filter, the fly's $\Delta \mathrm{LR}$ signal during each treatment was cross-correlated to the $\mathrm{m}$-sequence selected for that trial and then normalized to $\mid \mathrm{m}$-seq $\left.\right|^{2}$, as the mean value for the power spectral density of a white-noise sequence is expected to be the zeroth element of its autocorrelation function. This normalization results in units [V $\Delta \mathrm{LR} /(\mathrm{impulse} \cdot \mathrm{s})]$ and represents the gain of the linear filter, and since each impulse displaced the visual pattern in azimuth at $9750^{\circ} / \mathrm{s}$, the filter units are equivalent to (V $\Delta \mathrm{LR} /$ degree) adjusted by a constant factor. Therefore, the filter describes the change in the voltage of the $\Delta \mathrm{LR}$ signal per motion impulse per unit time. The mean filter was computed by averaging across individuals in each condition. For statistical purposes, the height of linear filters was assessed at a standard time point for each individual based on the peak of the mean filter. To reduce measurement error, the height of each individual's filter were also computed using the average value of a $40 \mathrm{~ms}$ time window centered on the peak of the mean impulse response function. Furthermore, the latency of the wild-type impulse response for each visual context was calculated as the average time required for the $\Delta$ LR signal to reach $0.005 \mathrm{~V}$ after $t=0$ (Fig. 2) regardless of olfactory context $(N=120$ for yaw, and $N=146$ for sideslip). Similarly, the time to peak was calculated as the mean time point of the maximum value attained by the filter for each visual context. Statistical significance in all cases was assessed with paired $t$ tests. All error bars plotted in the figures represent the $95 \%$ confidence interval as calculated from Student's $t$ distribution.

Predictions using the linear filters were generated by convolving the filter with the velocity of the desired stimulus. To calculate the predictions presented in Figure 3, the linear filter was scaled to reflect the fact that a single pixel step in the display corresponds to a $0.0521 \mathrm{~V}$ change on the display trace. The adjusted filters were convolved with the visual stimulus consisting of a sinusoidally modulated velocity profile presented at different oscillation frequencies (Fig. $3 A, B$, bottom) to predict the fly's behavior. To quantify how well the filters described the fly's response to the white noise stimulus, we calculated Pearson's correlation coefficient, $r$, given by the covariance of two signals normalized by the product of their SDs, between the fly's $\Delta$ LR response to the white noise stimulus and the prediction generated by the average linear filter for that condition.

\section{Results}

White noise analysis rapidly and accurately characterizes complex behavioral responses to wide-field motion

To characterize potentially subtle modulation of the OMR by olfactory stimulation or genetic circuit perturbation, we adopted a sensitive and robust quantitative behavioral assay. We presented yaw and sideslip optic flow fields to flies rigidly tethered to pins within an electronic visual flight simulator (Reiser and Dickinson, 2008) in combination with apple cider vinegar vapor, an attractive olfactory cue (Fig. $1 A-C$ ). Subsequently, we applied a white noise analysis technique (Fig. $1 D$ ) recently adapted to the virtual flight arena (Ringach and Shapley, 2004; Theobald et al., 2010a). Briefly, we measured the left-minus-right wingbeat amplitude $(\Delta \mathrm{LR})$, proportional to steering torque, while challenging animals with a white noise motion stimulus either in yaw or sideslip. By cross-correlating a white noise motion stimulus with the resulting steering response and dividing by the average value of the noise's power spectral density, we obtain the system's impulse response, $h(t)$. This function describes the fly's average turning effort following a brief motion step, but in a broader sense corresponds to the linear dependence of steering on continuous visual motion. White noise analysis assumes that the lumped system dynamics, which includes sensory input, neural computation, and motor output, is a mathematically linear timeinvariant dynamical system. In this case, linearity specifically encompasses two mathematical properties called superposition and homogeneity. Superposition requires that given two stimuli of magnitudes $s_{1}$ and $s_{2}$, the response to $s_{1}+s_{2}$ must equal the sum of the responses to the two stimuli separately. Similarly, homogeneity states that if the fly has a response $R$ to a stimulus $s_{1}$, and $s_{1}$ is scaled by a constant factor $a$, then the fly's response will be scaled by the same factor, resulting in a response $a \times R$. Additionally, the requirement for time invariance states that the system's response should not vary over time. Because of these properties, the impulse response correctly describes a linear system's response to any arbitrary stimulus over time (Fig. 1D). The predictive property of the impulse response arises simply because any arbitrary stimulus may be constructed as the sum of individual impulses and the response to the stimulus may be constructed as the sum of responses to those individual impulses. Do flies satisfy the assumptions of time invariance, superposition, and homogeneity?

These assumptions were tested by Theobald et al. (2010a) in the exact visual experimental paradigm used here. The study concluded that over the operational range of motor output in fly steering responses, the assumptions were satisfied to a surprising extent. In fact, the sideslip impulse response predicted $\sim 76 \%$ of the variance of the fly's measured response to a novel stimulus (Theobald et al., 2010a,b). White noise analysis allows rapid and accurate quantification of the impulse response to different types of optic flow, resulting in a recognizable transfer filter in just $N=1$ animal compared to $N=$ 364 through direct measurements of the animal's yaw response to motion impulses (Theobald et al., 2010a). The efficiency of the technique is particularly striking in sideslip-the impulse response becomes invariant after averaging the data from $\sim 10$ animals (our personal observation).

We used a pseudo-white noise binary "m-sequence" (maximum length shift register sequence) that efficiently covers all frequency components expected in a white noise stimulus within a significantly shorter time than randomly generated white noise (MacWilliams and Sloane, 1976; Ringach and Shapley, 2004). The $\mathrm{m}$-sequence consisted of $2^{n}-1$ binary digits, containing every possible combination of $n$ binary values. In our paradigm, the digit value +1 corresponded to a displacement of the visual panorama 1 pixel clockwise, and -1 a single step counterclockwise. Each single pixel displacement represents a motion impulse in our analysis (Fig. $1 E$ ). The sequence updated the visual display at $50 \mathrm{~Hz}$.

Armed with the impulse response, it is possible to predict the temporal dynamical response of the system to any arbitrary stimulus by convolving the impulse response with the stimulus (Fig. 1D). The m-sequence method produces a "sensorimotor filter" (Fig. $1 F$ ) that, when convolved with the $\mathrm{m}$-sequence stimulus (Fig. $1 E$ ), produces a prediction that closely matches the observed behavior (Fig. 1G). By plotting the normalized true response and prediction against each other, we may detect the presence of any static nonlinearities of the system as a deviation from a straight line intersecting the origin (Theobald et al., 2010a) (Fig. $1 H$ ). The high correlation value ( $r=0.82$, Pearson's correlation coefficient) suggests that the linear filter faithfully characterizes the dynamical properties of stabilization optomotor behavior to optic flow. 
A

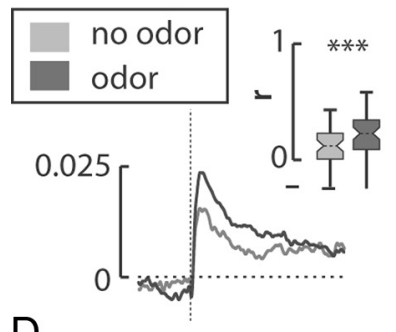

D

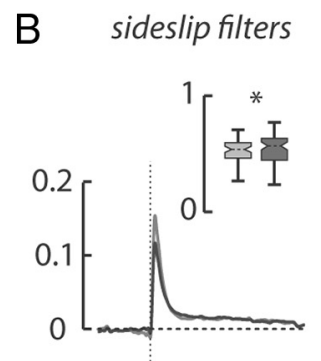

E

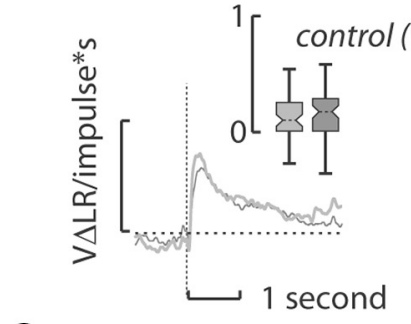

G

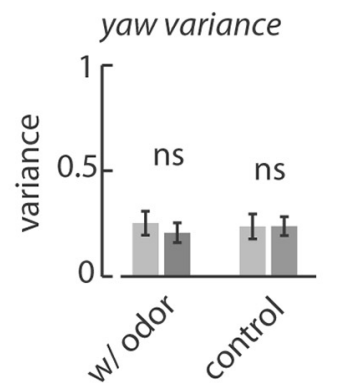

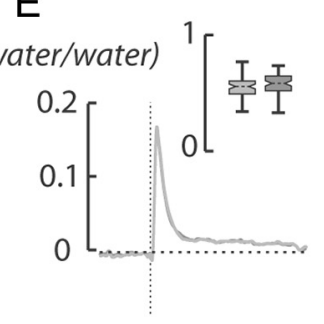

$\mathrm{H}$

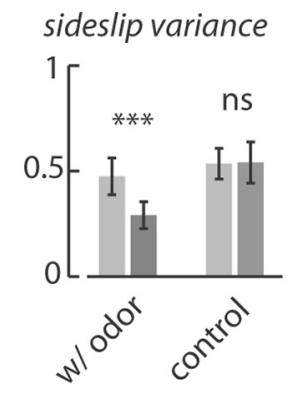

C

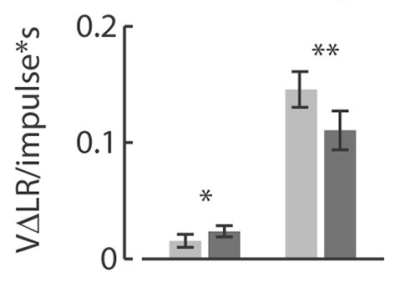

F

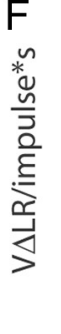

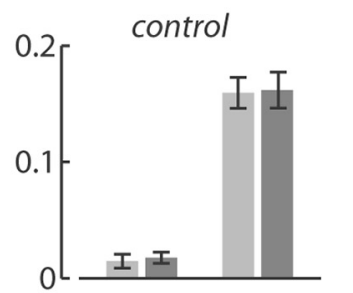

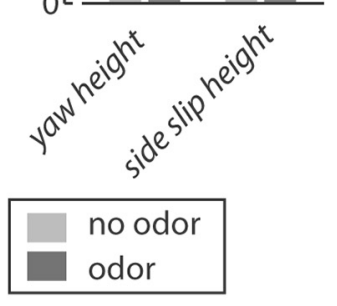

Figure 2. Olfactory cues modify the gain and reliability of the optomotor response. $\boldsymbol{A}, \boldsymbol{B}$, The yaw optomotor filter increases in magnitude (no odor $N=51, \operatorname{odor} N=58$ ) and in the prediction-response correlation (inset) due to the influence of odor (no odor gray, odor black) $(\boldsymbol{A})$, whereas the sideslip filter decreases in height but does not change in predictive power (no odor $N=66,0 \mathrm{dor}$ $N=71)(B)$. These changes in magnitude reflect changes in the gain of the filters in linear systems theory. $\boldsymbol{C}$, The measured changes in height at the peak of each filter ( $\sim 236$ ms for yaw and 109 ms for sideslip) differed significantly due to the influence of odor (paired $t$ test). $\boldsymbol{D}-\boldsymbol{F}$, In contrast, replacing the odor port with a second water vapor tube did not result in any qualitative changes in either the yaw (no odor $N_{1}=57$, no odor $N_{2}=62$ ) (D) or sideslip (no odor $N_{1}=75$, no odor $N_{2}=72$ ) $(\boldsymbol{E})$ filters or statistical differences in their peak heights $(\boldsymbol{F}), \mathbf{G}, \boldsymbol{H}$, Olfactory cues did not significantly increase the variance of the yaw steering response $(\boldsymbol{G})$ but did significantly decrease the variance of the sideslip steering response $(\boldsymbol{H})$. These changes in variance imply that the observed increase in filter gain due to odor is due to an increased steering effort correlated to the white noise stimulus $\left({ }^{*} p<\right.$ $\left.0.05,{ }^{* *} p<0.01,{ }^{* * *} p<0.001\right)$.

\section{Olfactory cues increase both the gain and fidelity of the yaw OMR and decrease the gain of the sideslip response}

We used the m-sequence method to derive behavioral impulse responses to two forms of optic flow, yaw and sideslip, in the presence and absence of odor. The impulse response can be thought of as a filter that describes the gain between a motion impulse and the fly's steering response over time. Filters tend to increase rapidly after the motion impulse occurring at a time $t=$ 0 , and after an onset latency specific to the visual context ( 37 and $83 \mathrm{~ms}$ for sideslip and yaw, respectively), rise rapidly, peak, and then decay slowly to a baseline value (Fig. $2 A, B$ ). A nonzero baseline after the decay indicates that the motion impulse has a persistent effect on the fly's steering attempt. Consistent with a previous study, sideslip filters show shorter onset latency and peak more rapidly and with greater magnitude than yaw filters. On average, the wild-type sideslip filter reached its peak value at $109 \mathrm{~ms}$ and the yaw filter reached its peak at $236 \mathrm{~ms}$. Olfactory cues significantly increase the height of yaw filters (Fig. $2 C, p<$ 0.05 , paired $t$ test) and decrease the height of sideslip filters $(p<$ 0.01 , paired $t$ test). These olfaction-mediated changes in filter gain are consistent with previous findings that attractive olfactory cues increase the amplitude of the yaw OMR and decrease the amplitude of the sideslip OMR to sinusoidal motion signals (Chow and Frye, 2008). To control for the potential influence of mechanosensory cues from small pressure surges during the odor-on switch, we replaced the apple cider vinegar with a second water tube. After the mechanical switch, there were no statistically significant differences in the magnitude of the filters (Fig. $2 D_{-}$ $F)$, confirming the specificity of optomotor gain enhancement to the olfactory stimulus.

Using the linear filter, we may directly quantify how much of the fly's behavior is explained by the optic flow stimulus by correlating the predictions made by the filter to the fly's actual response (or prediction-response correlation). We used the averaged population filters for the convolution operation (Fig. 2), as we assume that they represent a more accurate estimate of the "true" optomotor filter for each visual context than that measured from any individual animal. Olfactory cues increase the measured correlation coefficient $r$ of the yaw filter from 0.11 to 0.22 (Fig. $2 A$, inset, $p<0.001$, paired $t$ test) but do not significantly affect the correlation of the sideslip filter (Fig. $2 B$, inset, $r=0.51$ and 0.54 for no odor and odor, respectively). Switching to a second water-vapor stimulus similarly did not significantly change the prediction-response correlation (Fig. 2D,E).

We suspected that the olfactiondependent change in the height of yaw filter might also be related to the fidelity with which the fly transforms optic flow into motor output in addition to optomotor gain. The variance of the fly's response to the yaw m-sequence was not significantly increased by odor (Fig. 2G), which would appear to contradict the observed increase in the olfaction-dependent gain. However, the variance of the sideslip $\mathrm{m}$-sequence response decreases significantly with odor, which correlates with the olfaction-dependent attenuation of the sideslip filter (Fig. $2 H$ ). We hypothesize that in yaw this apparent conundrum may be explained by an influence of odor on the frequency of optomotor turns. That is, olfactory cues increase the fly's "attention" to optic flow cues, reducing the number of non-optomotor-induced turns, and therefore increasing both the prediction-response correlation and the height of the yaw filter. Together, these results show that olfactory cues differentially alter the gain of the sideslip and yaw filters, and also have a significant effect on the fidelity of the yaw optomotor response.

In a linear time-invariant dynamical system, the impulse response enables a prediction of the system's behavior to any arbitrary stimulus. To demonstrate this property, we convolved the impulse responses obtained in Figure $2 A$ with a sinusoidal motion stimulus (Fig. $3 A$, bottom) and compared the results to previously obtained data (Chow and Frye, 2008). The predictions reproduce many features of the actual data (Fig. $3 A, B$ ), including 
an increase in response amplitude in the yaw visual flow field under odor and a decrease in the amplitude of response in sideslip. However, the prediction misses the high-frequency cutoff present in both visual contexts (Fig. $3 A, B$, beginning at $\sim 9 \mathrm{~s}$ ). Whereas a dominant fraction of the fly's steering responses are linearly correlated to the visual stimulus, some responses are clearly not, such as the apparent rightward bias and the attenuation of response to high-frequency stimulus oscillation, both indicating nonlinearities in the flies' responses to our visual stimulus. To determine the phase relation of the predicted responses to the actual data, we plotted the data and predictions against each other (Fig. 3C,D). The distributions are largely colinear for each condition, with $r$ values near 0.84 for each treatment except for yaw without odor $(r=0.66)$. This shows that for yaw, the odor produces increased fidelity input-output behavior, whereas in sideslip, the sensorimotor transformation already functions at a highly reliable level independent of olfactory context.

\section{Olfactory modification of the yaw OMR is eliminated by larval hydroxyurea treatment}

We took a candidate approach in looking for the anatomical substrate of olfactiondependent changes in optomotor gain and fidelity. The fly MBs are best known for their role in olfactory learning and memory (Heisenberg, 2003). However, a growing body of literature also implicates them in multimodal sensory processing/context generalization (Liu et al., 1999), visual attention (van Swinderen and Greenspan, 2003; van Swinderen et al., 2009), and decision making (Tang and Guo, 2001; Zhang et al., 2007). By raising newly hatched Drosophila larvae in media infused with $50 \mathrm{mg} / \mathrm{ml} \mathrm{HU}$ for the first $5 \mathrm{~h}$ of life, we were able to prevent the development of the MBs (Prokop and Technau, 1994). To examine whether HU treatment compromised flight performance or olfaction generally, we measured the WBF of the animals at the beginning of each trial before and after the presentation of odor. Both wild-type and HU-treated animals maintain healthy WBF over $200 \mathrm{~Hz}$ preexposure, and subsequently exhibit the previously reported increase in WBF (Frye and Dickinson, 2004) in response to odor (Fig. 4A). In agreement with the WBF data and the wild-type results presented in Figure 2 , the variance of steering responses to the $\mathrm{m}$-sequence decreases significantly in sideslip ( $p<0.01$, paired $t$ test) but not in yaw with the addition of odor (Fig. $4 B$ ). Together, these results demonstrate that $\mathrm{HU}$-ablated animals respond rapidly and specifically to the onset of odor. HU-treated animals do not show statistically significant olfaction-dependent changes in gain for either yaw or sideslip (Fig. 4C) or significant odor-related changes in the prediction-response correlation (Fig. 4D), although a decreasing trend was apparent in the sideslip filter height (Fig. $4 C$, right, $p=0.25$ ). Although MB-ablated animals
B yaw

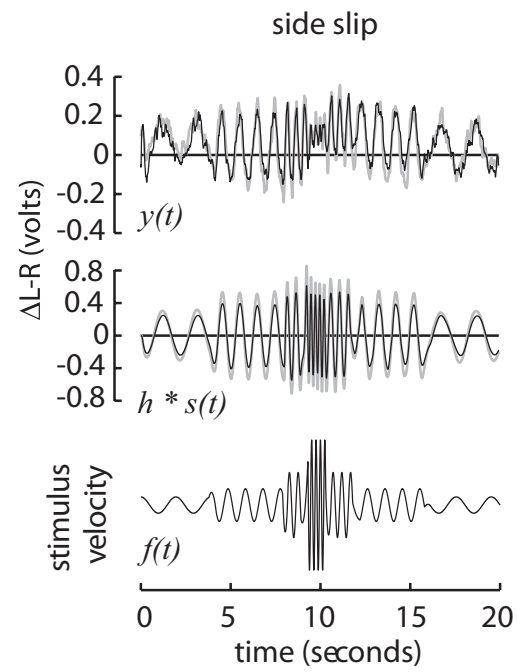

D

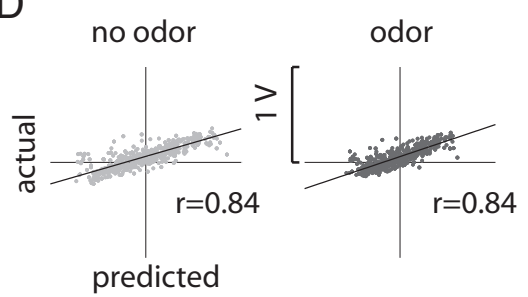

Figure 3. The prediction of the fly's steering response to a sinusoidal stimulus matches observed responses. A prediction was obtained by convolving the estimated filter with the stimulus velocity signal (see inset). $\boldsymbol{A}, \boldsymbol{B}$, In yaw, the prediction captures a previously observed increase in steering amplitude in response to odor $(\boldsymbol{A})$, and in sideslip, the prediction also matches a previously by Pearson's correlation coefficient, $r$, to a linear fit. $\boldsymbol{D}$, However, the phase correlation of the sideslip response is already largely independent of olfactory context.

have normal optomotor responses, they show a specific deficit in olfaction-dependent modification of these visual responses.

To build more evidence for the specificity of the HU treatment on olfactory modification of optomotor responses, we examined the ability of the flies to locate the source of an odor in a freechoice walking assay. HU-treated animals prefer odor-baited traps by comparison to water controls over a $24 \mathrm{~h}$ period, albeit with a slower time course than wild-type animals (Fig. 4E). The shift in time course may relate to a decreased olfactory behavioral sensitivity in MB-ablated animals during the challenging trap assay, in which odor gradients were established over time solely through diffusion. Nevertheless, MB-ablated animals still exhibit attraction to our odor and respond immediately to the onset of odor during flight. Together, these results suggest that modulatory circuits interacting with the yaw optomotor system require the presence of the MBs. Furthermore, the sideslip optomotor system retains an odor-dependent decrease in gain independent of the MBs, as evidenced by the decrease of response variance to the sideslip m-sequence and the decreasing trend in sideslip filter height.

To characterize the effect of losing OMOR, we convolved the HU filters with the same sinusoidal stimulus used previously (Fig. $3 A$ ). The predicted response of $\mathrm{HU}$ flies to olfactory cues have much lower amplitude than that of wild-type animals (Fig. $4 F$ ). These quantitative results indicate that a HU-defined circuit is indeed participating in the control dynamics for OMOR, and that 


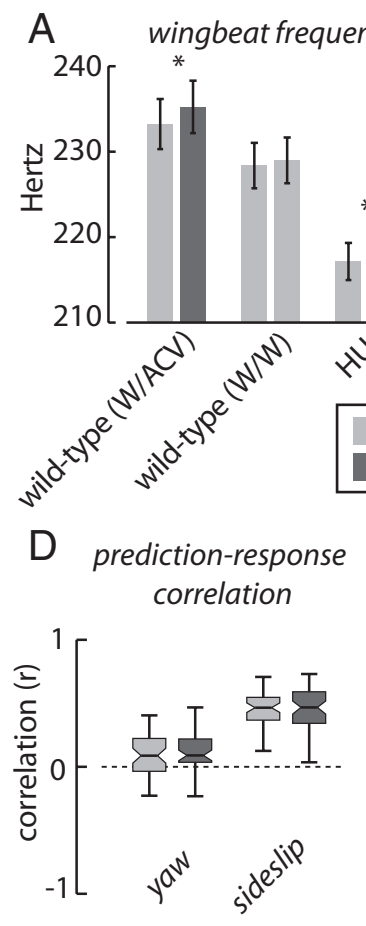

B HUvariance C

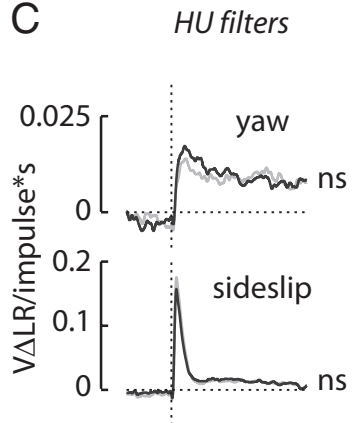

odor

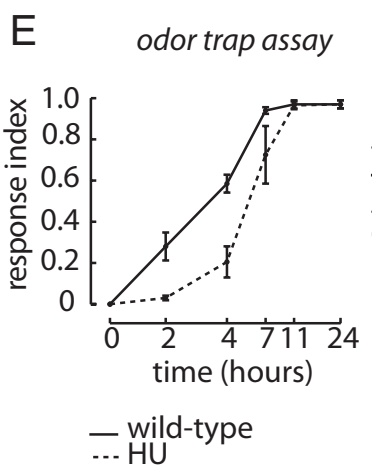

$\mathrm{F}$

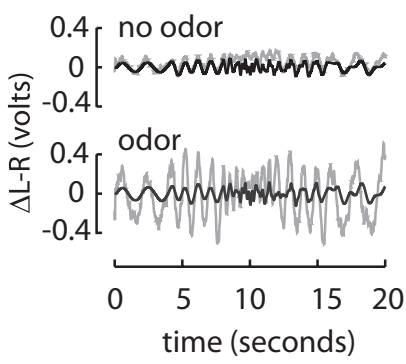

- predicted HU response - wild-type response

Figure 4. A circuit ablated by larval hydroxyurea treatment is required for yaw olfactory-mediated optomotor modification. Wild-type animals increase wingbeat frequency in the presence of an attractive odor, apple-cider vinegar (ACV), but not due to a switch to water stimuli (W). $\boldsymbol{A}, \boldsymbol{B}$, Similar to wild-type animals, HU-treated flies significantly increase their WBF in the presence of odor $(\boldsymbol{A})$ and decrease the variance of sideslip responses in the presence of odor $(\boldsymbol{B})$. $\boldsymbol{C}$, Treatment with HU results in a loss of olfaction-dependent changes in gain in both yaw and sideslip filters (yaw no-odor $N=49$, yaw odor $N=47$, sideslip no-odor $N=$ 57 , sideslip odor $N=59)$, although the sideslip height shows a decreasing trend $(p=0.25)$. $\boldsymbol{D}$, Neither filter shows olfactiondependent changes in prediction-response correlation. $\boldsymbol{E}$, Similar to wild-type animals, $\mathrm{HU}$-treated flies can localize an odor source hidden in the floor in a walking trap assay, albeit with a slower time course than wild-type animals. $\boldsymbol{F}$, The predicted yaw response to the sinusoidal stimulus in the presence of odor has much lower amplitude than the actual wild-type odor response. ${ }^{*} p<0.05$, ${ }^{* *} p<0.01,{ }^{* * *} p<0.001$

furthermore, this requirement is not due to a simple loss of the olfactory function, as $\mathrm{HU}$-treated flies retain some normal behavioral responses to odor.

\section{Genetic analysis implicates the MBs in OMOR but excludes specific MB subsets and the contribution of antennal lobe interneurons}

We attempted to replicate the loss of OMOR through the genetic inactivation of circuits defined by various gal4 lines via expression of TeTxLc or DT. Because HU treatment successfully eliminated OMOR, we chose a majority of lines that express primarily in the MBs. Altogether, 26 different strains of flies were tested. As expected, control lines expressing either TeTxLc alone (Fig. 5A, left) or wild-type animals of a non-CS strain (PCF) (Fig. 5A, right) exhibit wild-type yaw OMOR.

In contrast, lines with strong expression throughout the MBs, such as OK107-gal4 and c320-gal4, recapitulate the loss of OMOR when crossed with UAS-TnT (Fig. 5B). However, both lines have strong expression outside the MBs, notably in the second visual neuropil with OK107-gal4 and central complex with c320-gal4 (Aso et al., 2009). Interestingly, the yaw filters for each of these lines exhibits more transient dynamics than wild-type flies, returning to a zero baseline unlike wild-type yaw filters. However, expression of TeTxLc in the visual ganglia may only partially interfere with motion vision, as many motion-sensitive

neurons rely extensively on electrical synaptic connections (Borst et al., 2010), which are unaffected by TeTxLc. Expression of TeTxLc in more restricted $\mathrm{MB}$ lines has less apparent effects on OMOR. Crossing UAS-TnT to the lines c739-gal4 and $17 \mathrm{~d}$-gal4, largely specific to the $\alpha / \beta$ lobe of the MBs, the $\alpha^{\prime} / \beta^{\prime}$ line c305a-gal4, or the $\gamma$ lobe line H24-gal4 (Aso et al., 2009), had little effect on yaw OMOR (Fig. $5 C)$. However, expressing DT in these two lines resulted in a massive decrease in yaw filter gain (Fig. 5D). Aso et al. (2009) reports that $17 \mathrm{~d}, \mathrm{c} 739$, and MB247 have low to medium expression in the optic lobe ganglia. Therefore, the most parsimonious explanation for the loss of gain in yaw filters in the lines 17d-gal4; UAS-DTI and c739-gal4; UAS-DTI is the destruction of electrically coupled visual interneurons critical for yaw optic flow processing.

Although the reported expression patterns for the lines MB247-gal4 and D52Hgal4 are highly similar, affecting mainly the $\alpha / \beta$ and $\gamma$ lobes of the MBs (Aso et al., $2009)$, crossing them to UAS-TnT produced yaw filters with different shape (Fig. $5 E)$. The MB247 filter trajectory rises slowly, similar to the c739-gal4/UAS-DTI and $17 \mathrm{~d}$-gal4/UAS-DTI crosses, but still exhibits OMOR in contrast to D52H-gal4; UAS-TnT, which exhibits an OMOR deficit and rather high filter gain. We hypothesize that the loss of gain in the MB247 line may stem from the inactivation of a lobula plate circuit sensitive to TeTxLc and that the loss of OMOR in D52H-gal4 is the result of inactivating the same MB circuit ablated by the HU treatment. Why does the MB247-gal4 line not also exhibit a loss of OMOR? We speculate that subtle differences in the intensity and pattern of effector expression within the MBs between the two lines may result in the differential phenotypes.

Taken together, these effects indicate that eliminating MB function (in OK107-gal4 and c320-gal4) results in a loss of OMOR, but ablating function in only a MB subset, such as the $\alpha / \beta$ lobes (in c739-gal4 and $17 \mathrm{~d}$-gal4), the $\alpha^{\prime} / \beta^{\prime}$ lobes (c305agal4), or the $\gamma$ lobe (H24-gal4) does not. Furthermore, eliminating the function the $\alpha / \beta$ and $\gamma$ lobes simultaneously has variable results, resulting in a loss of OMOR in the case of $\mathrm{D} 52 \mathrm{H}$-gal4 but not MB247-gal4.

Although four progenitors ablated by $\mathrm{HU}$ treatment during the first $5 \mathrm{~h}$ of larval life develop specifically into the MB lobes (Ito and Hotta, 1992), a fifth neural progenitor develops into a set of local interneurons in the antennal lobe and a set of projection neurons (PNs) that connect the antennal lobe with both the MBs and the lateral horn (Stocker et al., 1997). The line GH298-gal4 specifically labels the antennal lobe lateral interneurons that are ablated by HU treatment. Although the reagent GH146-gal 4 labels the affected PNs, its broad expression in the optic ganglia unfortunately also eradicates behavioral sensitivity to optic flow (data not shown).

The more restrictive MB lines still exhibit OMOR, and therefore we examined whether the loss of olfactory modification 

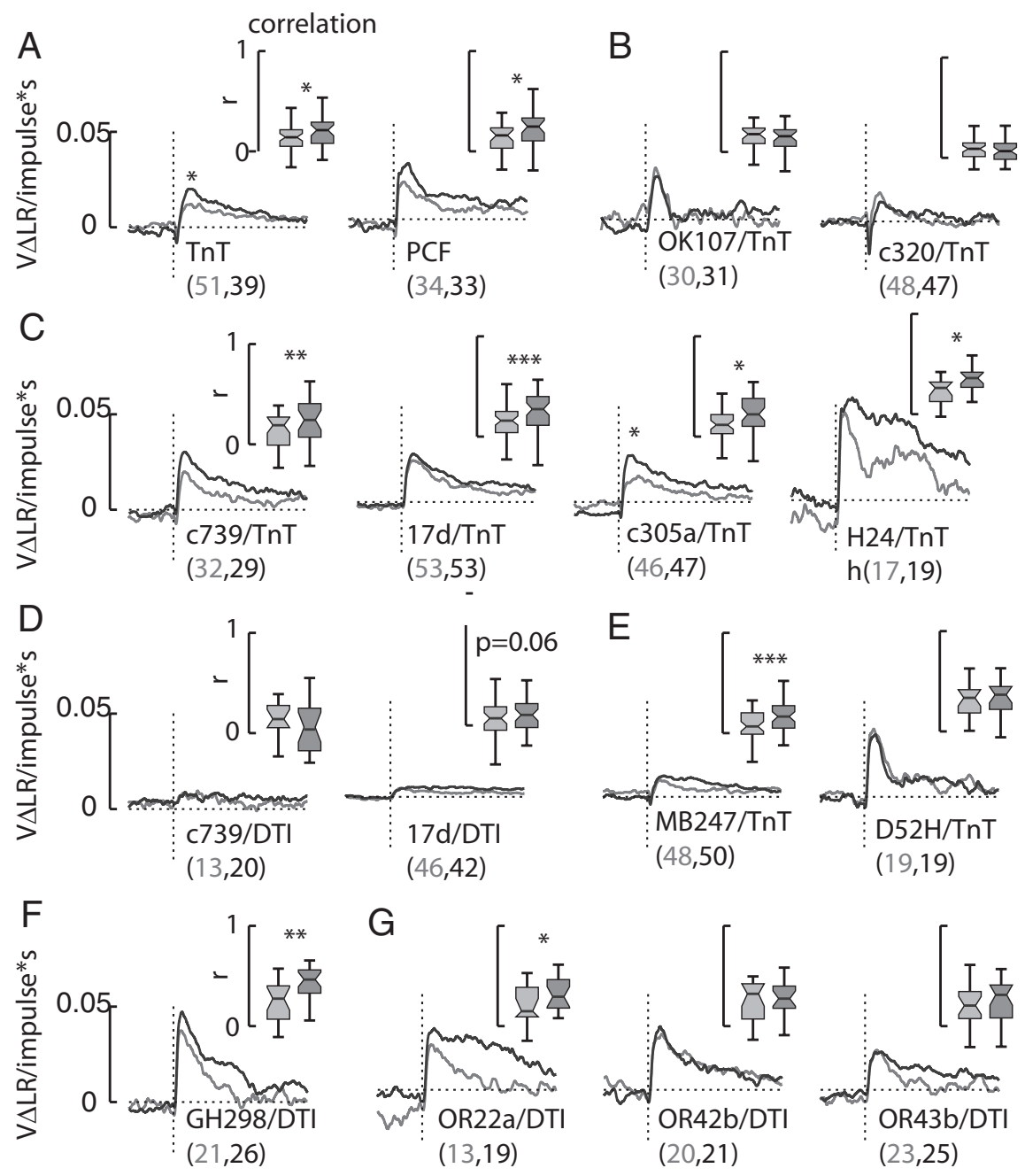

$\mathrm{H}$

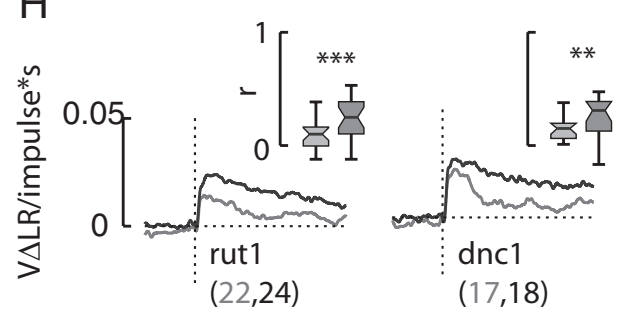

1 second
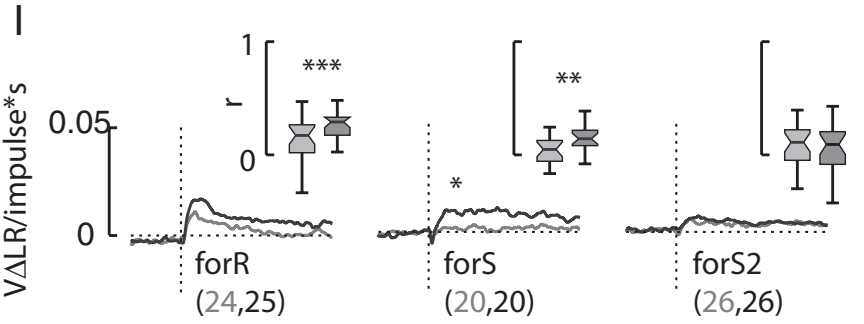

Figure 5. Transgenic yaw filters. $\boldsymbol{A}, \boldsymbol{B}$, Control lines UAS-TnT and PCF exhibit wild-type yaw OMOR $(\boldsymbol{A})$, whereas MB lines UAS-TnT $^{+/-} ; 0 \mathrm{~K} 107$-gal $4^{+/-}$and c320-gal4/UAS-TnT show a deficit in olfaction-dependent modulation of the yaw filters $(\boldsymbol{B})$. C, Expressing TeTxLc in the gal4-lines targeting single MB lobes, such as c739-gal4 $(\alpha / \beta), 17 \mathrm{~d}-\mathrm{gal} 4(\alpha / \beta)$, c305a-gal $4\left(\alpha^{\prime} / \beta^{\prime}\right)$, and H24-gal $4(\gamma)$, does not have a significant impact on 0MOR. D, Expressing DT with c739-gal4 and $17 \mathrm{~d}$-gal 4 results in animals with highly reduced yaw filters. $E$, Lines with wider MB expression, simultaneously targeting the $\alpha / \beta$ and $\gamma$ lobes, do not result in a loss of OMOR in the case of MB247-gal4 but do result in a loss of OMOR in the case of D52H-gal4. $F$, The line GH298-gal $4^{+/-}$; $\mathrm{UAS}-\mathrm{DTI}^{+/-}$exhibits robust OMOR, indicating that the antennal lobe local interneurons are not required. Similarly, expressing DT in the olfactory receptor neurons labeled by OR22a-gal 4 and OR43b-gal4 has no effect on OMOR. G, The line OR42b-gal ${ }^{+1-}$; $\mathrm{UAS}^{\mathrm{DTI}}{ }^{+/-}$exhibits a loss of OMOR. $\boldsymbol{H}$, Phosphodiesterase mutant dunce and adenylate cyclase mutant rutabaga both have normal yaw OMOR, as does the rover variant of the for allele. $I$, The sitter variant has very little OMR in the absence of odor and could be explained by compromised antennal lobe function rather than $\mathrm{MB}$ neuropil loss. The line GH298-gal4 ${ }^{+/-}$; UAS-DTI $^{+/-}$shows robust OMOR (Fig. $5 F$ ), indicating that not all local interneurons in the antennal lobe are required for olfaction-dependent changes in yaw filter gain or fidelity. Next, to confirm the specific olfactory receptor inputs to OMOR, we examined the effect of ablating olfactory receptor neurons OR22a, OR42b, and OR43b with UAS-DTI (Fig. 5G). Consistent with a study indicating that the DM1 glomerulus in the antennal lobe was necessary and sufficient for attraction to apple cider vinegar (Semmelhack and Wang, 2009), we observed a loss of OMOR in OR42 $\mathrm{b}^{+/-}$; UAS-DTI ${ }^{+/-}$but not in OR22a ${ }^{+/-}$; $\mathrm{UAS}^{-D T I}{ }^{+/-}$or $\mathrm{OR}_{43 \mathrm{~b}^{+/-}}$; UAS-DTI ${ }^{+/-}$. These results indicate that either the $\mathrm{PNs}$ ablated by $\mathrm{HU}$ treatment or the $\mathrm{MB}$ neuropil are required for OMOR.

Owing to the rich literature on learning and memory in the MBs, we also tested two olfactory learning mutants, $r u$ tabaga and dunce (mutations affecting the cAMP memory pathway), and three alleles of the for locus, encoding a protein kinase known to affect the propensity with which larvae distribute themselves on nutritive media (Pereira and Sokolowski, 1993). The cAMP mutants have wild-type yaw filters, albeit with prolonged dynamics in the presence of odor (Fig. $5 H$ ). Similarly the $R$ variant of the foraging locus also exhibits a wild-type yaw filter (Fig. $5 I$ ). However, the $S$ variant possesses very little yaw OMR in the absence of odor and produces a sharp step-like response in the presence of odor. Last, the S2 for variant shows a loss of OMOR and a reduced yaw OMR (Fig. 5I, far right). These results indicate that the OMR and OMOR are memory-independent interactions and thus were not much affected by mutations that disrupt olfactory learning and memory. However, the for locus fundamentally alters how flies react to visual motion and thus also affects visuomotor integration.

\section{Sideslip filters are relatively unaffected by $\mathrm{MB}$ inactivation}

In contrast to yaw filters, the sideslip filters were less affected by our genetic ma-

$\leftarrow$

the $\$ 2$ variant exhibits a large decrease in the magnitude of the yaw filter and a loss of 0MOR. ${ }^{*} p<0.05,{ }^{* *} p<0.01,{ }^{* * *} p<$ 0.001 . An asterisk over the filters indicates a significant difference in peak height. The numbers listed after the name of the line indicates the $N$ in the no-odor and odor conditions, respectively. 

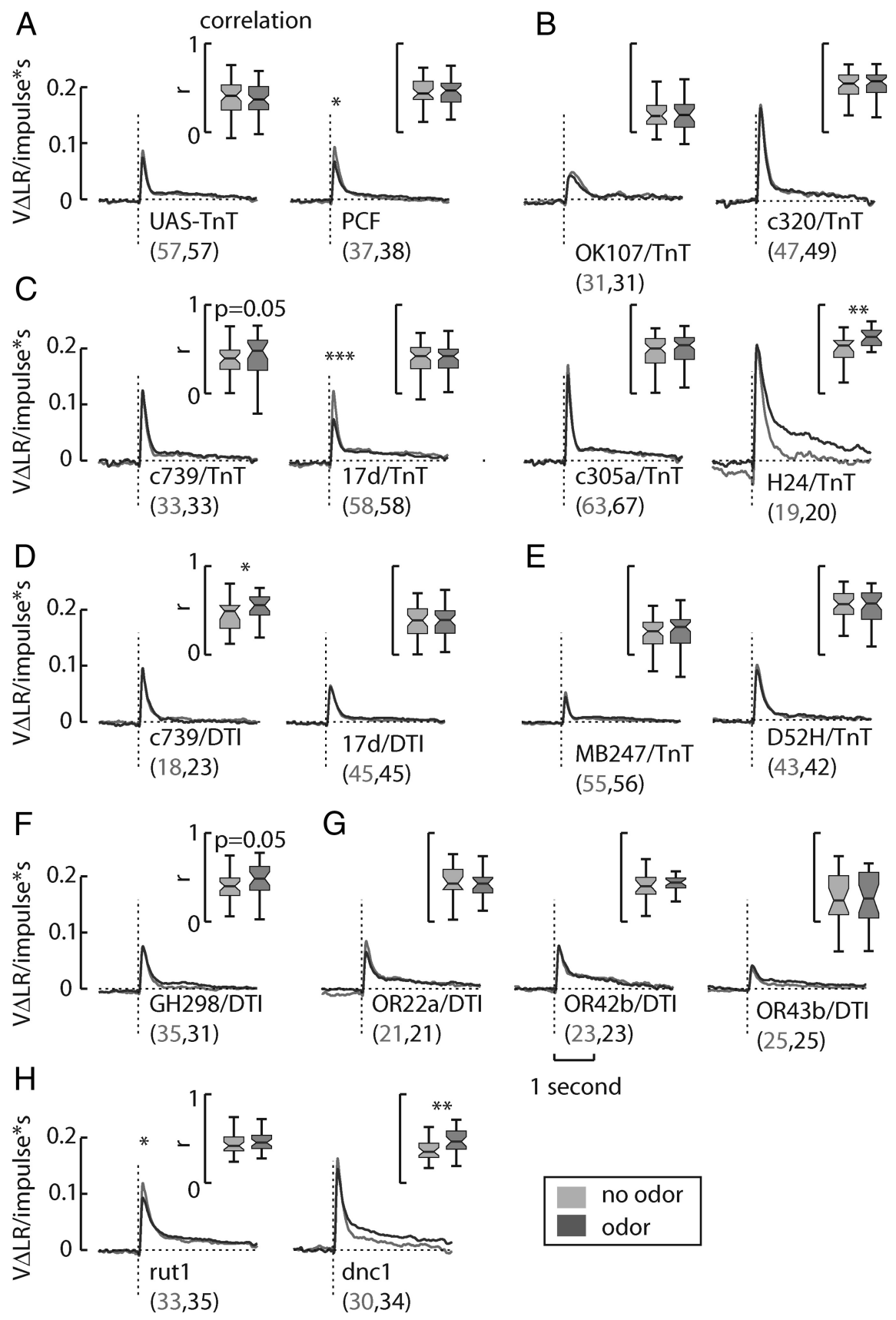

1 second
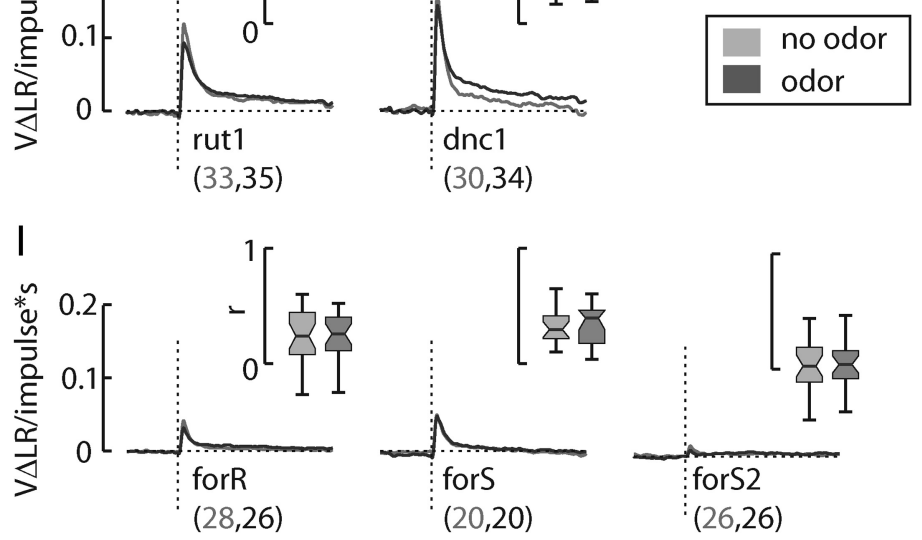

Figure 6. Transgenic sideslip filters. A, A statistically significant decrease in sideslip filter gain was not apparent in the control line UAS-TnT, although such a decrease was detected in the control line PCF. Both control sideslip filters exhibited wild-type dynamics. In contrast, the MB line UAS-TnT ${ }^{+/-} ; 0 \mathrm{~K} 107-\mathrm{gal} 4^{+/-}$had reduced sideslip filter with slower dynamics. $\boldsymbol{B}$, Since the MB line c320-gal4/UAS-TnT had a wild-type sideslip filter, the altered dynamics in the 0K107-gal4 line may be due to expression outside the MBs. Expressing TeTxLc in single MB lobes, such as in 7339 -gal4 $(\alpha / \beta), 17 \mathrm{~d}$-gal4 $(\alpha / \beta)$, c305a-gal4 $\left(\alpha^{\prime} / \beta^{\prime}\right)$, and H24-gal4 $(\gamma)$, did not much alter the overall dynamics of the sideslip response. C, The line 17d-gal4/UAS-TnT appeared to have an enhanced suppression of sideslip filter gain due to odor, and the line H24-gal4 seemed to exhibit an inverted response to odor-an increase of sideslip filter gain. $\boldsymbol{D}$, Expressing DT with $\mathrm{c} 739$-gal 4 and $17 \mathrm{~d}$-gal4 did not much affect the sideslip filter, by comparison to its effects on the respective yaw filters. $E$, However, the $\alpha / \beta$ and $\gamma$ lobes line UAS-TnT ${ }^{+/-} ;$MB247-gal $4^{+/-}$had nipulations, confirming both the overall "health" of the transgenic flies and also the specificity of the manipulation of visuoolfactory integration. Both control lines, UAS-TnT and PCF, broadly exhibit the same response dynamics as the wild-type CS line, although UAS-TnT does not show a significant decrease in filter gain due to the influence of odor (Fig. 6A). The line OK107-gal4/UAS-TnT (Fig. 6B, left) exhibits a decrease in filter gain and altered response dynamics similar to its yaw filter, owing perhaps to the expression of TeTxLc in the visual system. In contrast to its yaw filter, the sideslip filter in c320gal4/UAS-TnT is wild type save for a lack of olfactory modulation (Fig. $6 B$, right). However, since the control line UASTnT also fails to exhibit an olfactiondependent decrease in sideslip filter gain, we cannot conclusively attribute this to the intended genetic manipulation. Similarly, the lobe-specific MB lines (see previous section for expression pattern) c739-gal4/UASTnT, c305a-gal4/UAS-TnT (Fig. 6C), c739gal4; UAS-DTI (Fig. 6D), and D52H-gal4; UAS-TnT (Fig. 6E) all lack olfactiondependent decreases in sideslip gain similar to the control line UAS-TnT but are otherwise wild type. However, the $\alpha / \beta$ line $17 \mathrm{~d}$ gal4; UAS-DTI (Fig. $6 D$ ) and the $\alpha / \beta$ and $\gamma$ line MB247-gal4/UAS-TnT (Fig. 6E) do show decreased sideslip filter gain, presumably, as in the case of their respective yaw filters, owing to expression in key motion-sensitive neurons in the optic ganglia. Finally, the line $\alpha / \beta$ line 17d-gal4/UAS-TnT (Fig. 6C) exhibits a robust olfaction-dependent decrease in the height of its sideslip filter (perhaps even more than observed in the wild-type filter) and the $\gamma$ line UAS-TnT; H24-gal4 (Fig. 6C, top right) appears to have a reversed olfactory influence, with odor increasing the gain of the sideslip filter. Together, these results suggest that inactivating the MBs has little effect on the sideslip optomotor response. However, it may be possible to alter the

\section{$\leftarrow$}

a greatly decreased sideslip filter, perhaps due to expression in the optic ganglia, while the line D52H-gal4; UAS-TnT was wild type. $\boldsymbol{F}$, Neither the antennal lobe interneuron line GH298$\mathrm{gal}^{+/-} ; \mathrm{UAS}^{\mathrm{DTI}}{ }^{+/-}$or lines expressing DT in $\mathrm{OR}$ lines $22 \mathrm{a}$ or $42 \mathrm{~b}$ had abnormal sideslip filters. $\mathbf{G}$, The line 0 R43bgal $4^{+/-} ;$UAS-DTI ${ }^{+/-}$inexplicably had reduced filter gain. $H, I$, cAMP mutants rut 1 and $d n c 1$ both had wild-type sideslip filters $(\boldsymbol{H})$, while for lines $R$, $S$, and $S 2$ all had highly reduced sideslip filters $(\boldsymbol{I}) .{ }^{*} p<0.05,{ }^{* *} p<0.01,{ }^{* * *} p<0.001$. An asterisk over the filters indicates a significant difference in peak height. The numbers listed after the name of the line indicates the $N$ in the no odor and odor conditions, respectively. 
modulation of the response to odor through inactivation of certain lobes, either enhancing the wild-type effect, as with $17 \mathrm{~d}$-gal4, or perhaps even reversing it, as with $\mathrm{H} 24$-gal4.

We similarly observed little impact on the sideslip filter through expression of DT in the peripheral olfactory lines GH298-gal4 (Fig. 6F), OR22a-gal4, and OR42b-gal4 (Fig. 6G). However, expression of DT in OR43b-gal4 resulted in a low-gain sideslip filter (Fig. 6G). We speculate that changes in filter gain are more subtle in sideslip than in yaw and thus may have escaped detection with the number of flies tested in these lines (Chow and Frye, 2008).

Finally, both olfactory learning mutants rut1 and $d n c 1$ exhibit wild-type sideslip filters, although $d n c$ responds differently to odor than wild-type animals, increasing its prediction-response correlation in the presence of odor and lowering filter gain after the response peak (Fig. $6 H$ ). All three for lines showed decreased sideslip filter gain, with an almost nonexistent response in forS2 (Fig. 6I), which highlights that genetic background has a sizeable influence on the response dynamics of the flies.

\section{Yaw and sideslip filters are genetically separable}

We observed several lines that exhibit a degree of genetic separability between yaw and sideslip filters. Although expression of TeTxLc in the $\alpha / \beta$ line 739 -gal4 and in the $\alpha / \beta$ and $\gamma$ line 201y-gal4 resulted in roughly wild-type yaw and sideslip filters (Fig. $7 A, B$ ), crossing these lines with UAS-DTI produced either a large decrease in yaw filter gain (c739-gal4 Fig. 7C, but also see 17d-gal4, Figs. 5, 6C) or a highly aberrant yaw filter (Fig. 7D) without much altering the gain of their respective sideslip filters. Crossing these lines to MBGAL80 improved their yaw filters without rescuing OMOR (Fig. $7 E, F$ ). These results most likely indicate that $\mathrm{c739-}$ gal4 and 201y-gal4 impinge on a motion-sensitive neuron in the optic lobes that contributes strongly to yaw optomotor behavior. The overall improvement of yaw filter shape may suggest that the MBs may boost the output of the motion-detection system downstream of the optic ganglia. Furthermore, the loss of OMOR despite blocking expression of DT in the MBs suggests that the output of the affected circuit is required for olfaction to have its influence on motion detection in yaw.

\section{Discussion}

In this study, we apply a systems identification technique using white noise to derive linear filters that formalize the dynamical transformation of optic flow into flight stabilization responses (Fig. 1). Under normal flight conditions, flies depend upon visual feedback to localize the source of an odor during flight (Frye et al., 2003), which they use to maintain flight heading in a plume (Duistermars and Frye, 2008). Here we demonstrate an olfaction-dependent increase in the gain of yaw optomotor transformations and a decrease in the gain of sideslip transformations (Figs. 2, 3). These changes to the fly's flight control algorithm would stabilize forward flight heading up an odor plume by rejecting unintended deviations in rotation resulting in straight flight, which is a hallmark of odor tracking in free flight (Budick and Dickinson, 2006). We next demonstrate that the $\mathrm{MB}$ circuits ablated by larval $\mathrm{HU}$ treatment are required for olfactory modification of the optomotor reflex (Fig. 4). An analysis of transgenic flies expressing circuit inactivators TeTxLc or DT in spatially defined neural subsets suggests redundancy in multimodal MB circuits, as only inactivation of the entire $\mathrm{MB}$ proved completely effective in replicating the HU ablation phenotype (Fig. 5). Furthermore, the optomotor phenotypes derived through white noise analysis are genetically separable for yaw and sideslip, and underscore partially parallel sensory-motor processing pathways in motion-processing circuits handling these types of optic flow (Fig. 5). Finally, the learning and memory cAMP mutants dunce and rutabaga do not exhibit defects in optomotor modification, although they have prolonged optomotor dynamics (Figs. 6, 7). Therefore, a MB-dependent olfactory circuit mediates a fast-acting learning independent modulation in the visuomotor transformation pathway (Fig. 8). 


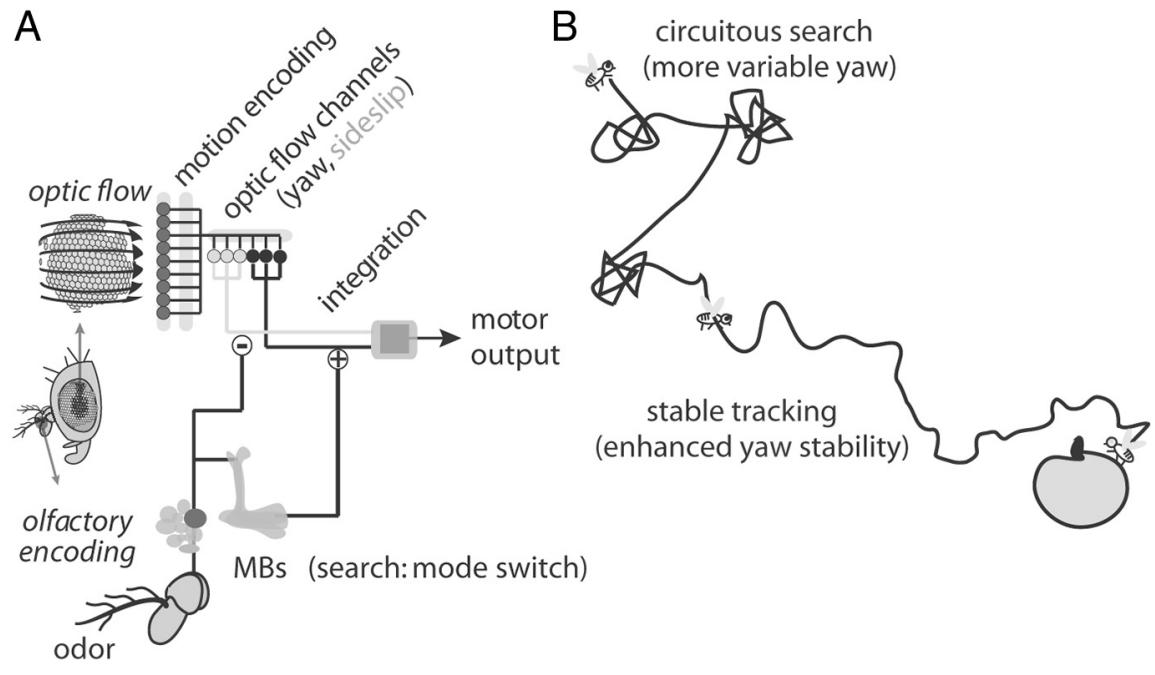

Figure 8. A hypothesized circuit for olfactory modification of the optomotor reflex. $A$, We posit that yaw and sideslip optomotor responses rely on different networks of electrically coupled LPTCs, or at least functionally separated parallel visual-motor control channels. Olfactory cues influence the two optomotor channels disparately, increasing gain in the yaw motor transformation and reducing gain in sideslip. Furthermore, $\mathrm{HU}$ ablation defines the olfactory circuit responsible for olfaction-dependent optomotor modification, suggesting the involvement of the MBs. $\boldsymbol{B}, 0$ lfactory information acts as a switch between a search state that allows greater flexibility in the yaw optomotor channel and tracking state that requires greater yaw fidelity to track odor plumes.

in manipulations of high-order visual circuitry (Katsov and Clandinin, 2008).

\section{Olfactory input gates yaw optomotor fidelity}

We observed an olfaction-dependent increase in yaw gain. Similarly, we show that olfactory input increases the fidelity of the optomotor transformation. Recently, it has been demonstrated that active movement increases the gain of HS and VS LPTC responses (Chiappe et al., 2010; Maimon et al., 2010; Rosner et al., 2010). However, given that animals in our study were flying in both olfactory contexts, and that changes in LPTC gain did not correspond to changes in head optomotor gain (Rosner et al., 2010), we can reasonably exclude this possibility from explaining the specificity of changes to yaw responses. Furthermore, theoretical calculations imply that the LPTCs encode sensory stimuli more reliably than the head optomotor response would predict (Warzecha et al., 2000).

Multimodal influence on descending

\section{Circuit divisions in the processing of sideslip and yaw}

Optic flow is encoded as early as the third optic ganglion, where $\sim 60$ lobula plate tangential cells (LPTCs) respond to motion in a preferred direction with receptive fields reminiscent of roll movements (VS cells) or yaw movements (HS cells) during flight (Borst et al., 2010). The LPTCs involved in processing rotation and translation necessarily must pool information from both eyes. Indeed, contralateral axo-axonal electrical coupling to HSE confers yaw optic flow selectivity over forward translation to $\mathrm{H} 2$ (Farrow et al., 2006). Neurons immediately downstream, such as the descending neurons of the ocellar and vertical system (Wertz et al., 2009), may generate more reliable representations of motion through electrical coupling of the LPTCs. Similarly, some neck motor neurons generate binocular visual receptive fields by pooling across LPTCs from the two hemispheres (Huston and Krapp, 2008). By contrast to yaw, there is no known singular LPTC that acts as a tuned spatial filter for sideslip optic flow (Borst and Weber, 2011). Nevertheless, investigators report that HS cells, sensitive to horizontal motion, are also sensitive to sideslip (Kern et al., 2005) as well as to vertical motion (Haag and Borst, 2003). It is likely that sideslip is encoded by combining the output of several different types of LPTCs. Binocular electrical coupling through $\mathrm{H} 1$ and $\mathrm{H} 2$ result in the particular sensitivity pattern of HSE (Krapp et al., 2001), and many gap junctions have been identified to play critical roles in the downstream processing of LPTC output (Huston and Krapp, 2008, 2009; Wertz et al., 2009; Haag et al., 2010).

Notably, many of our genetic manipulations have independent effects on the yaw and sideslip filters (but see OK107-gal4/ UAS-TnT and $17 \mathrm{~d}$-gal4/UAS-TnT). In extreme cases, it is possible to completely disrupt the yaw filter without affecting the sideslip filter (in DT lines c739, and 201y, Fig. 8). Interestingly, disrupted yaw filters were only apparent with the use of DT, corroborating the role that electrical synapses play in the yaw optomotor response. Our results would suggest that the motion pathways encoding yaw and sideslip are susceptible to genetic dissection. Similar genetically separable effects on yaw and translation elicited behavioral responses have been observed motor output has been demonstrated in a number of systems. For example, in moths, pheromones gate the amplitude of premotor descending afferent responses (Olberg and Willis, 1990). In Drosophila, the timing of efferent motor output onto asynchronous steering muscles during flight is regulated by mechanosensory signals to coincide with the proper phase of the wingbeat cycle (Tu and Dickinson, 1996). Furthermore, fly neck motor neurons, which are active during gaze stabilization, require mechanosensory input as well as a central flight signal to produce suprathreshold responses to motion (Huston and Krapp, 2009; Haag et al., 2010, Frye, 2010). Here we show that at least in Drosophila, an attractive food odorant also modifies visually mediated motor output.

\section{Are the mushroom bodies required for olfactory modification of the optomotor reflex?}

Our results demonstrate circuits disabled by hydroxyurea treatment are required for OMOR. This treatment specifically ablates four mushroom body neuroblasts and a lateral neuroblast resulting in the complete loss of the MBs, a local interneuron circuit in the antennal lobe, and PNs that contact the MB calyces via the lateral horn on the inner antenno-cerebral tract (Ito and Hotta, 1992; Stocker et al., 1997). Genetic analysis of the optomotor filters using expression of TeTxLc in the MB lines OK107-gal4 and c320-gal4 lends support to our interpretation that the MBs are required for OMOR. Interestingly, attempting to inactivate MB subsets with c739-gal4, 17d-gal4, c305a-gal4, H24-gal4, and MB247-gal4 did not recapitulate the optomotor phenotype observed with HU ablation. However, D52H-gal4 exhibited a loss of OMOR with TeTxLc expression. Together, these results suggest that the MBs have some functional redundancy with regards to olfaction-dependent modification of the yaw and sideslip filters. Therefore, one must eliminate the function of the entire MB lobe to provoke a loss of olfaction-dependent optomotor modification.

We are able to exclude the local interneuron line defined by GH298-gal4 from consideration as a subset of the OMOR circuit (Fig. 5F), leaving only the subset of PNs and the MBs as candidates. However, the PN line ablated by HU is unlikely to be in- 
volved in mediating behavioral attraction to food odorants. The antennal lobe glomerulus DM1 has been shown to be both necessary and sufficient for attraction to apple cider vinegar, the odorant used in the present study (Semmelhack and Wang, 2009). Indeed, expressing DT in OR $42 \mathrm{~b}$ receptor neurons, which primarily innervate DM1, results in the loss of yaw OMOR, whereas expression in OR22a and OR43b receptor neurons did not (Fig. 5G). Interestingly, the HU-ablated PNs synapse exclusively to a single glomerulus in the antennal lobe (Stocker et al., 1997). Although the glomerulus was not identified by Stocker et al. (1997), given the fact that the PNs are derived from the lateral neuroblast and synapse to a central location within the antennal lobe, the most likely candidates are VC2 and VA7M (Jefferis et al., 2001). Furthermore, the anterodorsal cluster of neuroblasts giving rise to PNs that contribute to more dorsal glomeruli was not ablated by HU treatment (Stocker et al., 1997). Despite the ablation of thousands of olfactory neurons by $\mathrm{HU}$ treatment, flies still respond quickly and specifically to the onset of odor (Fig. $5 A, B$ ), which indicates that the loss of OMOR was not due to an inability to detect the odorant. Furthermore, our results using the odor trap assay suggest that the attraction circuit was not disrupted, as walking animals were still trapped with virtually maximal efficacy with apple cider vinegar (Fig. 5D). Together, our findings suggest that OMOR circuits are separate from circuits responsible for attraction to food odorants and the loss of OMOR in HU ablated animals was not due to a loss of olfactory sensitivity. Our study shows that almost paradoxically, visual input into the MBs is not required in order for the MBs to participate in visuo-olfactory integration. Instead, olfactory information seems to act as a switch, changing a noisy sensorimotor transformation into a reliable one.

\section{References}

Aso Y, Grübel K, Busch S, Friedrich AB, Siwanowicz I, Tanimoto H (2009) The mushroom body of adult Drosophila characterized by GAL4 drivers. J Neurogenet 23:156-172.

Borst A, Weber F (2011) Neural action fields for optic flow based navigation: a simulation study of the fly lobula plate network. PLoS ONE 6:e16303.

Borst A, Haag J, Reiff DF (2010) Fly motion vision. Annu Rev Neurosci 33:49-70.

Budick SA, Dickinson MH (2006) Free-flight responses of Drosophila melanogaster to attractive odors. J Exp Biol 209:3001-3017.

Chiappe ME, Seelig JD, Reiser MB, Jayaraman V (2010) Walking modulates speed sensitivity in Drosophila motion vision. Curr Biol 20:1470-1475.

Chow DM, Frye MA (2008) Context-dependent olfactory enhancement of optomotor flight control in Drosophila. J Exp Biol 211:2478-2485.

Chow DM, Frye MA (2009) The neuro-ecology of resource localization in Drosophila: behavioral components of perception and search. Fly (Austin) 3:50-61.

Davis RL (2011) Traces of Drosophila memory. Neuron 70:8-19.

Duistermars BJ, Frye MA (2008) Crossmodal visual input for odor tracking during fly flight. Curr Biol 18:270-275.

Duistermars BJ, Chow DM, Condro M, Frye MA (2007) The spatial, temporal and contrast properties of expansion and rotation flight optomotor responses in Drosophila. J Exp Biol 210:3218-3227.

Farrow K, Haag J, Borst A (2006) Nonlinear, binocular interactions underlying flow field selectivity of a motion-sensitive neuron. Nat Neurosci 9:1312-1320.

Frye MA (2010) Multisensory systems integration for high-performance motion control in flies. Curr Opin Neurobiol 20:347-352.

Frye MA, Dickinson MH (2004) Motor output reflects the linear superposition of visual and olfactory inputs in Drosophila. J Exp Biol 207:123-131.

Frye MA, Tarsitano M, Dickinson MH (2003) Odor localization requires visual feedback during free flight in Drosophila melanogaster. J Exp Biol 206:843-855.

Götz KG (1964) Optomotorische Untersuchung des visuellen systems einiger Augenmutanten der Fruchtfliege Drosophila. Biol Cybern 2:77-92.
Haag J, Borst A (2003) Orientation tuning of motion-sensitive neurons shaped by vertical-horizontal network interactions. J Comp Physiol A Neuroethol Sens Neural Behav Physiol 189:363-370.

Haag J, Wertz A, Borst A (2010) Central gating of fly optomotor response. Proc Natl Acad Sci U S A 107:20104-20109.

Hassenstein B, Reichardt W (1956) Systemtheoretische analyse der zeit-, reihenfolgen-, und vorzeichenauswertung bei der bewgungsperzeption des russelkafers Chlorophanus. Z Naturforsch B 11:513-524.

Heisenberg M (2003) Mushroom body memoir: from maps to models. Nat Rev Neurosci 4:266-275.

Holmes TC, Sheeba V, Mizrak D, Rubovszky B, Dahdal D (2007) Circuitbreaking and behavioral analysis by molecular genetic manipulation of neural activity in Drosophila. In: Invertebrate neurobiology (North G, ed), pp 19-52. Cold Spring Harbor: Cold Spring Harbor Laboratory.

Huston SJ, Krapp HG (2008) Visuomotor transformation in the fly gaze stabilization system. PLoS Biol 6:e173.

Huston SJ, Krapp HG (2009) Nonlinear integration of visual and haltere inputs in fly neck motor neurons. J Neurosci 29:13097-13105.

Ito K, Awasaki T (2008) Clonal unit architecture of the adult fly brain. Adv Exp Med Biol 628:137-158.

Ito K, Hotta Y (1992) Proliferation pattern of postembryonic neuroblasts in the brain of Drosophila melanogaster. Dev Biol 149:134-148.

Jefferis GS, Marin EC, Stocker RF, Luo L (2001) Target neuron prespecification in the olfactory map of Drosophila. Nature 414:204-208.

Katsov AY, Clandinin TR (2008) Motion processing streams in Drosophila are behaviorally specialized. Neuron 59:322-335.

Kern R, van Hateren JH, Michaelis C, Lindemann JP, Egelhaaf M (2005) Function of a fly motion-sensitive neuron matches eye movements during free flight. PLoS Biol 3:e171.

Krapp HG, Hengstenberg R, Egelhaaf M (2001) Binocular contributions to optic flow processing in the fly visual system. J Neurophysiol 85:724-734. Land MF (1997) Visual acuity in insects. Annu Rev Entomol 42:147-177.

Lee T, Lee A, Luo L (1999) Development of the Drosophila mushroom bodies: sequential generation of three distinct types of neurons from a neuroblast. Development 126:4065-4076.

Liu L, Wolf R, Ernst R, Heisenberg M (1999) Context generalization in Drosophila visual learning requires the mushroom bodies. Nature 400:753-756.

MacWilliams FJ, Sloane NJA (1976) Pseudo-random sequences and arrays. Proc IEEE 64:1715-1729.

Maimon G, Straw AD, Dickinson MH (2010) Active flight increases the gain of visual motion processing in Drosophila. Nat Neurosci 13:393-399.

Margulies C, Tully T, Dubnau J (2005) Deconstructing memory in Drosophila. Curr Biol 15:R700-R713.

Olberg RM, Willis MA (1990) Pheromone-modulated optomotor response in male gypsy moths, Lymantria dispar L-directionally selective visual interneurons in the ventral nerve cord. J Comp Physiol A Neuroethol Sens Neural Behav Physiol 167:707-714.

Pereira HS, Sokolowski MB (1993) Mutations in the larval foraging gene affect adult locomotory behavior after feeding in Drosophila melanogaster. Proc Natl Acad Sci U S A 90:5044-5046.

Prokop A, Technau GM (1994) Normal function of the mushroom body defect gene of Drosophila is required for the regulation of the number and proliferation of neuroblasts. Dev Biol 161:321-337.

Reiser MB, Dickinson MH (2008) A modular display system for insect behavioral neuroscience. J Neurosci Methods 167:127-139.

Reynolds AM, Frye MA (2007) Free-flight odor tracking in Drosophila is consistent with an optimal intermittent scale-free search. PLoS ONE 2:e354.

Ringach D, Shapley R (2004) Reverse correlation in neurophysiology. Cogn Sci 28:147-166.

Rosner R, Egelhaaf M, Warzecha AK (2010) Behavioural state affects motion-sensitive neurones in the fly visual system. J Exp Biol 213:331-338.

Semmelhack JL, Wang JW (2009) Select Drosophila glomeruli mediate innate olfactory attraction and aversion. Nature 459:218-223.

Stebbings LA, Todman MG, Phillips R, Greer CE, Tam J, Phelan P, Jacobs K, Bacon JP, Davies JA (2002) Gap junctions in Drosophila: developmental expression of the entire innexin gene family. Mech Dev 113:197-205.

Stocker RF, Heimbeck G, Gendre N, de Belle JS (1997) Neuroblast ablation in Drosophila P[GAL4] lines reveals origins of olfactory interneurons. J Neurobiol 32:443-456. 
Tammero LF, Frye MA, Dickinson MH (2004) Spatial organization of visuomotor reflexes in Drosophila. J Exp Biol 207:113-122.

Tang S, Guo A (2001) Choice behavior of Drosophila facing contradictory visual cues. Science 294:1543-1547.

Theobald JC, Ringach DL, Frye MA (2010a) Dynamics of optomotor responses in Drosophila to perturbations in optic flow. J Exp Biol 213: $1366-1375$.

Theobald JC, Ringach DL, Frye MA (2010b) Visual stabilization dynamics are enhanced by standing flight velocity. Biol Lett 6:410-413.

Tu MS, Dickinson MH (1996) The control of wing kinematics by two steering muscles of the blowfly (Calliphora vicina). J Comp Physiol A 178:813-830.

van Swinderen B, Greenspan RJ (2003) Salience modulates 20-30 Hz brain activity in Drosophila. Nat Neurosci 6:579-586. van Swinderen B, McCartney A, Kauffman S, Flores K, Agrawal K, Wagner J, Paulk A (2009) Shared visual attention and memory systems in the Drosophila brain. PLoS One 4:e5989.

Warzecha AK, Kretzberg J, Egelhaaf M (2000) Reliability of a fly motionsensitive neuron depends on stimulus parameters. J Neurosci 20: $8886-8896$.

Wertz A, Gaub B, Plett J, Haag J, Borst A (2009) Robust coding of egomotion in descending neurons of the fly. J Neurosci 29:14993-15000.

Xi W, Peng Y, Guo J, Ye Y, Zhang K, Yu F, Guo A (2008) Mushroom bodies modulate salience-based selective fixation behavior in Drosophila. Eur J Neurosci 27:1441-1451.

Zhang K, Guo JZ, Peng Y, Xi W, Guo A (2007) Dopamine-mushroom body circuit regulates saliency-based decision-making in Drosophila. Science 316:1901-1904. 